\title{
Anomalous dimensions from Yukawa couplings in SMNEFT: four-fermion operators
}

\author{
Alakabha Datta, ${ }^{a}$ Jacky Kumar, ${ }^{b}$ Hongkai Liu ${ }^{c}$ and Danny Marfatia ${ }^{d}$ \\ ${ }^{a}$ Department of Physics and Astronomy, University of Mississippi, \\ Oxford, MS 38677, U.S.A. \\ ${ }^{b}$ Institute for Advanced Study, Technical University Munich, \\ 85748 Garching, Germany \\ ${ }^{c}$ Department of Physics and Astronomy, University of Pittsburgh, \\ Pittsburgh, PA 15260, U.S.A. \\ ${ }^{d}$ Department of Physics and Astronomy, University of Hawaii at Manoa, \\ Honolulu, HI 96822, U.S.A. \\ E-mail: datta@phy.olemiss.edu, jacky.kumar@tum.de, hol42@pitt.edu, \\ dmarf8@hawaii.edu
}

AbSTRACT: The Standard Model Neutrino Effective Field Theory (SMNEFT) is the Standard Model Effective Field Theory (SMEFT) augmented with right-handed neutrinos. Building on our previous work, arXiv:2010.12109, we calculate the Yukawa coupling contributions to the one-loop anomalous dimension matrix for the 11 dimension-six four-fermion SMNEFT operators. We also present the new contributions to the anomalous dimension matrix for the 14 four-fermion SMEFT operators that mix with the SMNEFT operators through the Yukawa couplings of the right-handed neutrinos.

Keywords: Beyond Standard Model, Effective Field Theories, Neutrino Physics

ArXiv EPrint: 2103.04441 


\section{Contents}

1 Introduction 1

2 Formalism 2

2.1 Wavefunction renormalization 3

2.2 Counterterms 4

3 Results 6

3.1 Anomalous dimensions from Yukawa couplings: SMNEFT 6

$3.1 .1 \quad(\bar{R} R)(\bar{R} R) \quad 6$

$3.1 .2 \quad(\bar{L} L)(\bar{R} R) \quad 7$

3.1.3 $(\bar{L} R)(\bar{R} L)$ and $(\bar{L} R)(\bar{L} R) \quad 8$

3.2 Anomalous dimensions from Yukawa couplings: SMEFT 9

$3.2 .1 \quad(\bar{L} L)(\bar{L} L) \quad 9$

$\begin{array}{lll}3.2 .2 & (\bar{L} L)(\bar{R} R) & 10\end{array}$

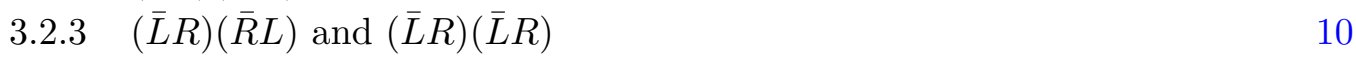

4 Summary 10

\section{Introduction}

The sterile right-handed neutrino is one of the most well studied extensions of the Standard Model (SM) motivated by, among other things, the observation of neutrino masses and mixing. Instead of considering all possible models, an efficient alternative is to use a model independent approach based on the principles of effective theory. The idea is to construct all possible operators representing the interactions of sterile neutrinos with SM fields consistent with the symmetries of the SM. The framework is valid between the scale of electroweak symmetry breaking, $\mu_{E W}$, and the cut-off scale for new physics, $\Lambda$.

In the effective theory approach, the leading terms of the effective Lagrangian are given by the SM, and the new interactions of the right-handed neutrino with the SM fields are described by higher dimension operators,

$$
\mathcal{L}=\sum_{i} \mathcal{C}_{i} \mathcal{O}_{i}
$$

The operators $\mathcal{O}_{i}$ respect the $\mathrm{SU}(3)_{C} \times \mathrm{SU}(2)_{L} \times \mathrm{U}(1)_{Y}$ gauge symmetry and are constructed from SM and right-handed neutrino fields. The renormalization scale dependent Wilson coefficient $(\mathrm{WC}) \mathcal{C}_{i}$, determines the size of the contribution of operator $\mathcal{O}_{i}$, and is calculated by matching the effective theory with the underlying theory. 
Given the absence of new physics signals at the LHC, the use of effective theory to study physics beyond the SM has received much attention recently. With only SM fields, the Standard Model Effective Field Theory (SMEFT) is obtained [1-4], and the one-loop renormalization group evolution (RGE) of all dimension-six operators have been presented in refs. [5-7].

Extending SMEFT with sterile right-handed neutrinos $n$, yields the Standard Model Neutrino Effective Field Theory (SMNEFT) [8-12]. Loop effects in SMNEFT have only recently started being studied. We presented the gauge terms of the one-loop RGE of all dimension-six operators in SMNEFT [13]. The mixing between the bosonic operators was discussed in ref. [14], and the one-loop RGE of a subset of four-fermion operators was provided in ref. [15].

In this paper we calculate the one-loop RGE of all dimension-six four-fermion SMNEFT operators that arises from the Yukawa interactions of the Higgs, right-handed neutrino and SM fields. We assume the neutrinos are Dirac in nature. Our formalism is generalizable to the Type-I two-Higgs-doublet model. Note that the neutrino Yukawa couplings may be large if a contribution to Dirac neutrino masses from high scale physics, as for example in ref. [16], is rendered small by virtue of a cancellation by the Yukawa contribution.

We present our calculations and results in the gauge basis because a transformation to the mass basis involves a rotation of the quark and lepton fields to their mass eigenstates. This rotation is inherently model-dependent since only the left-handed quark and lepton mixing matrices are experimentally accessible. Moreover, how the Yukawa interactions of the right-handed neutrinos relate to the neutrino mixing parameters depends on the mechanism of neutrino mass generation.

The paper is organized as follows. In section 2 , we introduce the formalism to compute the RGE of the dimension-six operators. In section 3, we present the one-loop anomalous dimension matrix (ADM) of all four-fermion operators in SMNEFT, and the additional RGE terms in SMEFT that arise from Yukawa couplings of the right-handed neutrinos. Finally, in section 4 we present our summary.

\section{Formalism}

The SMNEFT Lagrangian is

$$
\mathscr{L}_{\text {SMNEFT }} \supset i \bar{n} \not \partial n+\mathscr{L}_{\text {Yukawa }}+\sum_{i} \mathcal{C}_{i} \mathcal{O}_{i},
$$

where $\mathcal{C}_{i}$ are the WCs of the dimension-six operators. The Yukawa terms with generation indices suppressed, are

$$
\mathscr{L}_{\text {Yukawa }}=-\left[\phi^{\dagger j} \bar{d} Y_{d} q_{j}+\tilde{\phi}^{\dagger j} \bar{u} Y_{u} q_{j}+\phi^{\dagger j} \bar{e} Y_{e} \ell_{j}+\tilde{\phi}^{\dagger j} \bar{n} Y_{n} \ell_{j}+\text { h.c. }\right]
$$

where $\phi$ is the Higgs doublet and $\tilde{\phi}^{j}=\epsilon^{j k} \phi_{k}^{*}$. The four types of Yukawa interaction vertices for the quark sector are shown in figure 1 .

There are a total of $16(\Delta B=0=\Delta L)$ new operators in the SMNEFT framework, which are shown in table 1 in the Warsaw basis convention [2]. 


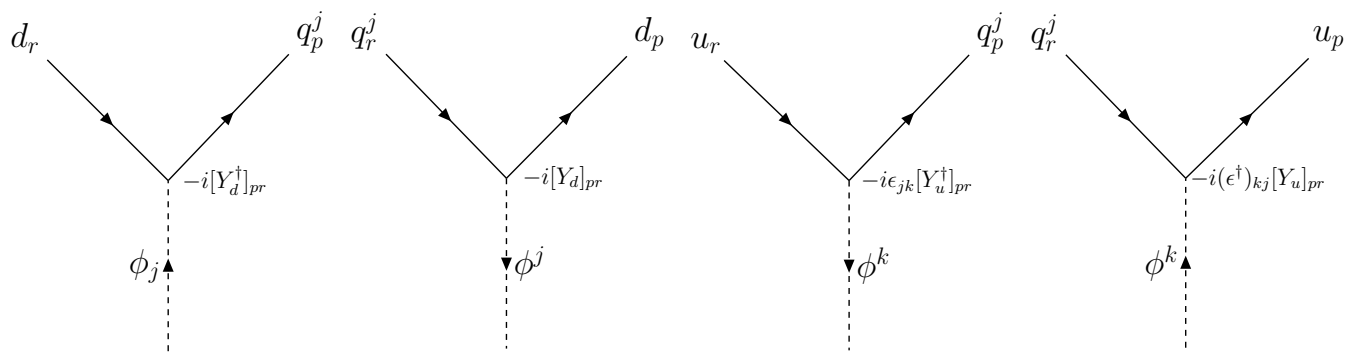

Figure 1. The four types of Yukawa interaction vertices. The flavor indices ' $p r$ ' and $\mathrm{SU}(2)_{L}$ indices ' $j k$ ' are written explicitly.

\begin{tabular}{|c|c|c|c|c|c|}
\hline \multicolumn{2}{|c|}{$(\bar{R} R)(\bar{R} R)$} & \multicolumn{2}{|c|}{$(\bar{L} L)(\bar{R} R)$} & \multicolumn{2}{|c|}{$(\bar{L} R)(\bar{R} L)$ and $(\bar{L} R)(\bar{L} R)$} \\
\hline $\mathcal{O}_{n d}$ & $\left(\bar{n}_{p} \gamma_{\mu} n_{r}\right)\left(\bar{d}_{s} \gamma^{\mu} d_{t}\right)$ & $\mathcal{O}_{q n}$ & $\left(\bar{q}_{p} \gamma_{\mu} q_{r}\right)\left(\bar{n}_{s} \gamma^{\mu} n_{t}\right)$ & $\mathcal{O}_{\ell n \ell e}$ & $\left(\bar{\ell}_{p}^{j} n_{r}\right) \epsilon_{j k}\left(\bar{\ell}_{s}^{k} e_{t}\right)$ \\
$\mathcal{O}_{n u}$ & $\left(\bar{n}_{p} \gamma_{\mu} n_{r}\right)\left(\bar{u}_{s} \gamma^{\mu} u_{t}\right)$ & $\mathcal{O}_{\ell n}$ & $\left(\bar{\ell}_{p} \gamma_{\mu} \ell_{r}\right)\left(\bar{n}_{s} \gamma^{\mu} n_{t}\right)$ & $\mathcal{O}_{\ell n q d}^{(1)}$ & $\left(\bar{\ell}_{p}^{j} n_{r}\right) \epsilon_{j k}\left(\bar{q}_{s}^{k} d_{t}\right)$ \\
$\mathcal{O}_{n e}$ & $\left(\bar{n}_{p} \gamma_{\mu} n_{r}\right)\left(\bar{e}_{s} \gamma^{\mu} e_{t}\right)$ & & & $\mathcal{O}_{\ell n q d}^{(3)}$ & $\left(\bar{\ell}_{p}^{j} \sigma_{\mu \nu} n_{r}\right) \epsilon_{j k}\left(\bar{q}_{s}^{k} \sigma^{\mu \nu} d_{t}\right)$ \\
$\mathcal{O}_{n n}$ & $\left(\bar{n}_{p} \gamma_{\mu} n_{r}\right)\left(\bar{n}_{s} \gamma^{\mu} n_{t}\right)$ & & & $\mathcal{O}_{\ell n u q}$ & $\left(\bar{\ell}_{p}^{j} n_{r}\right)\left(\bar{u}_{s} q_{t}^{j}\right)$ \\
$\mathcal{O}_{n e d u}$ & $\left(\bar{n}_{p} \gamma_{\mu} e_{r}\right)\left(\bar{d}_{s} \gamma^{\mu} u_{t}\right)$ & & & & \\
\hline \multicolumn{2}{|c|}{$\psi^{2} \phi^{3}$} & & $\psi^{2} \phi^{2} D$ & $\psi^{2} X \phi$ \\
\hline $\mathcal{O}_{n \phi}$ & $\left(\phi^{\dagger} \phi\right)\left(\bar{l}_{p} n_{r} \tilde{\phi}\right)$ & $\mathcal{O}_{\phi n}$ & $i\left(\phi^{\dagger} \stackrel{\leftrightarrow}{D}_{\mu} \phi\right)\left(\bar{n}_{p} \gamma^{\mu} n_{r}\right)$ & $\mathcal{O}_{n W}$ & $\left(\bar{\ell}_{p} \sigma^{\mu \nu} n_{r}\right) \tau^{I} \tilde{\phi} W_{\mu \nu}^{I}$ \\
& & $\mathcal{O}_{\phi n e}$ & $i\left(\tilde{\phi}^{\dagger} D_{\mu} \phi\right)\left(\bar{n}_{p} \gamma^{\mu} e_{r}\right)$ & $\mathcal{O}_{n B}$ & $\left(\bar{\ell}_{p} \sigma^{\mu \nu} n_{r}\right) \tilde{\phi} B_{\mu \nu}$ \\
\hline
\end{tabular}

Table 1. The 16 SMNEFT operators involving the right-handed neutrinos $n$ in the Warsaw convention which conserve baryon and lepton number $(\Delta B=\Delta L=0)$. The flavor indices 'prst' are suppressed for simplicity. The fundamental $\mathrm{SU}(2)_{L}$ indices are denoted by $j, k$, and $I$ is the adjoint index.

The Lagrangian can be written in terms of bare fields $\overrightarrow{\mathcal{O}}^{(0)}$ as

$$
\mathscr{L}_{\text {SMNEFT }} \supset \overrightarrow{\mathcal{C}}^{T} \cdot \overrightarrow{\mathcal{O}}+\text { counterterms } \equiv \overrightarrow{\mathcal{C}}^{T} \cdot \mathbf{Z} \cdot \overrightarrow{\mathcal{O}}^{(0)},
$$

where $\mathbf{Z}=\mathbf{Z}_{\mathrm{ct}} / \mathbf{Z}_{\mathrm{wr}}$ is the renormalization constant matrix which depends on corrections from the counterterms, $\mathbf{Z}_{\mathrm{ct}}$, and the wavefunction renormalizations, $\mathbf{Z}_{\mathrm{wr}}$. Given that the bare operators and Lagrangian are independent of the renormalization scale $\mu$, the RG equations for the Wilson coefficients are

$$
\dot{\overrightarrow{\mathcal{C}}} \equiv 16 \pi^{2} \mu \frac{d}{d \mu} \overrightarrow{\mathcal{C}}=-16 \pi^{2}\left(\mathbf{Z}^{T}\right)^{-1} \mu \frac{d}{d \mu} \mathbf{Z}^{T} \overrightarrow{\mathcal{C}} .
$$

The main task is to calculate the expressions for $\mathbf{Z}_{\mathrm{wr}}$ and $\mathbf{Z}_{\mathrm{ct}}$, which is detailed below.

\subsection{Wavefunction renormalization}

The bare field $\psi^{(0)}$ is related to the renormalized field $\psi^{R}$ via

$$
\psi^{R}=\frac{1}{\sqrt{Z_{\psi}}} \psi^{(0)} .
$$




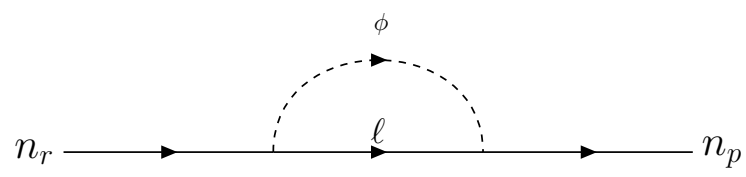

Figure 2. Self-energy of right-handed neutrino $n$.

For the four-fermion operator, $\mathcal{O}_{4 \psi}=\bar{\psi}_{1} \psi_{2} \bar{\psi}_{3} \psi_{4}$, the wavefunction renormalization constant is given by

$$
\mathbf{z}_{\mathrm{wr}} \equiv \sqrt{\prod_{i=1}^{4} \mathbf{z}_{\psi_{i}}} .
$$

From figure 2, the Yukawa-dependent wavefunction renormalization of a right-handed neutrino $n$ is

$$
Z_{n}^{(Y)}=1-\frac{\substack{p r \\ p r}_{p r}^{(Y)}}{16 \pi^{2} \epsilon},
$$

where $\underset{p r}{\gamma_{n}(Y)}=\left[Y_{n} Y_{n}^{\dagger}\right]_{p r}$, in the notation of ref. [6]. We have taken dimension, $D=4-2 \epsilon$, in dimensional regularization. Similarly, we have,

$$
\begin{aligned}
& \gamma_{\substack{\ell \\
\gamma^{\prime}}}^{(Y)}=\frac{1}{2}\left[Y_{e}^{\dagger} Y_{e}+Y_{n}^{\dagger} Y_{n}\right]_{p r}, \quad \gamma_{p r}^{(Y)}=\left[Y_{e} Y_{e}^{\dagger}\right]_{p r}, \\
& \underset{p r}{\gamma_{q}^{(Y)}}=\frac{1}{2}\left[Y_{d}^{\dagger} Y_{d}+Y_{u}^{\dagger} Y_{u}\right]_{p r}, \quad \gamma_{p r}^{(Y)}=\left[Y_{d} Y_{d}^{\dagger}\right]_{p r}, \quad \underset{p r}{\gamma_{u}^{(Y)}}=\left[Y_{u} Y_{u}^{\dagger}\right]_{p r}
\end{aligned}
$$

\subsection{Counterterms}

The corrections from counterterms cancel the ultraviolet (UV) divergence from the oneloop diagrams. In the one-loop diagrams, there are 14 different structures as in figure 3; there are seven counterparts to those shown. We display the UV divergent part of each structure in figure 3.

The UV divergent parts in figures 3(a) to 3(c) are of the form

$$
\begin{aligned}
& \mathcal{D}_{a}=-\frac{1}{64 \pi^{2} \epsilon}\left(\bar{\psi}_{1} \gamma^{\mu} \Gamma_{1} \gamma_{\mu} \psi_{2}\right)\left(\psi_{3} \Gamma_{2} \psi_{4}\right), \\
& \mathcal{D}_{b}=-\frac{1}{64 \pi^{2} \epsilon}\left(\bar{\psi}_{1} \gamma^{\mu} \Gamma_{1} \psi_{2}\right)\left(\bar{\psi}_{3} \gamma_{\mu} \Gamma_{2} \psi_{4}\right), \\
& \mathcal{D}_{c}=-\frac{1}{64 \pi^{2} \epsilon}\left(\bar{\psi}_{1} \Gamma_{1} \gamma^{\mu} \psi_{2}\right)\left(\bar{\psi}_{3} \gamma_{\mu} \Gamma_{2} \psi_{4}\right),
\end{aligned}
$$

where $\Gamma_{1}$ and $\Gamma_{2}$ are the Lorentz structures for the upper and lower vertex, respectively. In figure $3(\mathrm{~d}), \Gamma_{1}$ has to be $P_{2}$, which is the projection operator of the chiral fermion field $\psi_{2}$, because for the other possibilities, the UV divergent parts vanish. Thus we obtain

$$
\mathcal{D}_{d}=\frac{1}{16 \pi^{2} \epsilon}\left(\bar{\psi}_{1} P_{2} \psi_{2}\right)\left(\bar{\psi}_{3} P_{4} \psi_{4}\right) .
$$

The UV divergent part of figure 3(e) is of the form

$$
\mathcal{D}_{e}=-\frac{1}{32 \pi^{2} \epsilon}\left(\bar{\psi}_{1} \gamma^{\mu} P_{2} \psi_{2}\right)\left(\bar{\psi}_{3} \gamma_{\mu} P_{4} \psi_{4}\right) \text {. }
$$




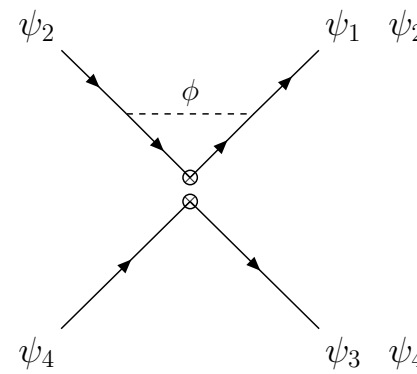

(a)

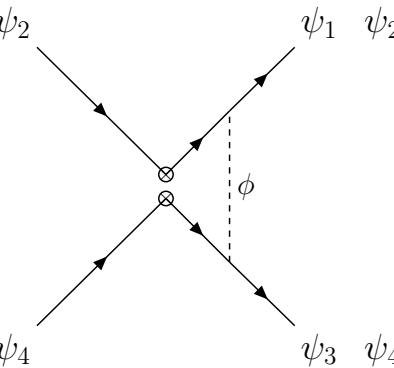

(b)

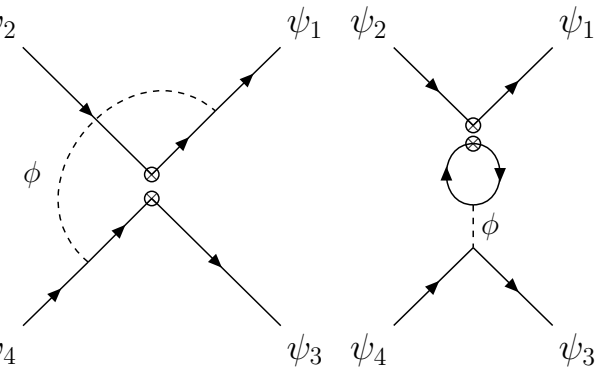

(c) (d)

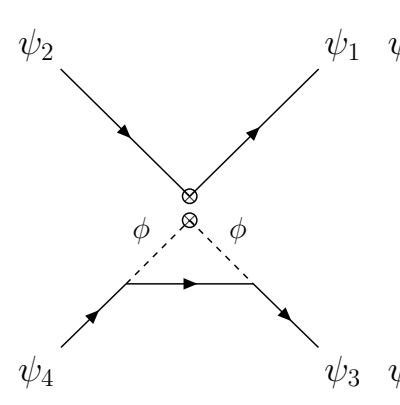

(e)

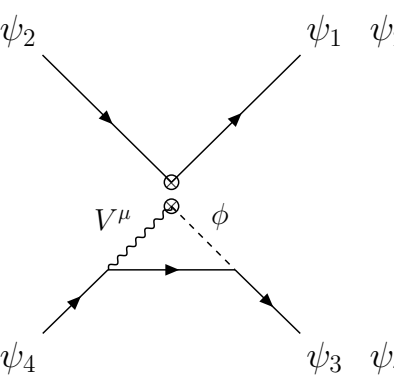

(f)

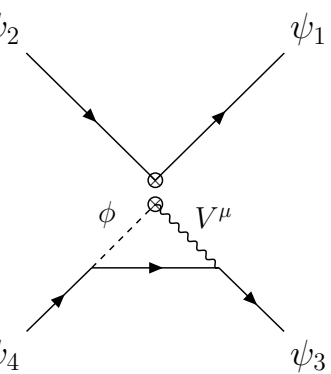

(g)

Figure 3. The seven structures that contribute to the four-fermion operator anomalous dimension matrix at the one-loop level. The $\psi_{i}$ are operator-dependent external fermions. The fermion inside the loop is related to $\psi_{i}$ through Yukawa or gauge couplings.

For the dipole operators in figures $3(\mathrm{f})$ and $3(\mathrm{~g})$, the UV divergent parts are of the form

$$
\begin{aligned}
\mathcal{D}_{f} & =\frac{i}{64 \pi^{2} \epsilon}\left(\bar{\psi}_{1} \sigma^{\mu \nu} P_{2} \psi_{2}\right)\left(\bar{\psi}_{3} \gamma^{\beta} \gamma^{\alpha} P_{4} \psi_{4}\right)\left(g^{\mu \beta} g^{\nu \alpha}-g^{\mu \alpha} g^{\nu \beta}\right) \\
& =\frac{1}{32 \pi^{2} \epsilon}\left(\bar{\psi}_{1} \sigma^{\mu \nu} P_{2} \psi_{2}\right)\left(\bar{\psi}_{3} \sigma_{\mu \nu} P_{4} \psi_{4}\right), \\
\mathcal{D}_{g} & =\frac{i}{64 \pi^{2} \epsilon}\left(\bar{\psi}_{1} \sigma^{\mu \nu} P_{2} \psi_{2}\right)\left(\bar{\psi}_{3} \gamma^{\alpha} \gamma^{\beta} P_{4} \psi_{4}\right)\left(g^{\mu \beta} g^{\nu \alpha}-g^{\mu \alpha} g^{\nu \beta}\right) \\
& =-\frac{1}{32 \pi^{2} \epsilon}\left(\bar{\psi}_{1} \sigma^{\mu \nu} P_{2} \psi_{2}\right)\left(\bar{\psi}_{3} \sigma_{\mu \nu} P_{4} \psi_{4}\right) .
\end{aligned}
$$

To simplify our results further, we follow ref. [6] and define the amplitudes in figure 4 in connection with figure $3(\mathrm{~d})$ :

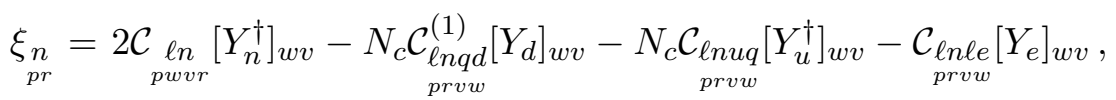

$$
\begin{aligned}
& \underset{p r}{\xi_{e}}=2 \mathcal{C}_{p w e}^{\ell e}\left[Y_{e}^{\dagger}\right]_{w v}-N_{c} \mathcal{C}_{\ell e d q}\left[Y_{d}^{\dagger}\right]_{w v}+\underset{\text { prvw }}{N_{c}} \mathcal{C}_{\text {lequ }}^{(1)}\left[Y_{u}\right]_{w v}-\underset{\text { prvw }}{\mathcal{C}_{\text {lnepr }}}\left[Y_{n}\right]_{w v},
\end{aligned}
$$

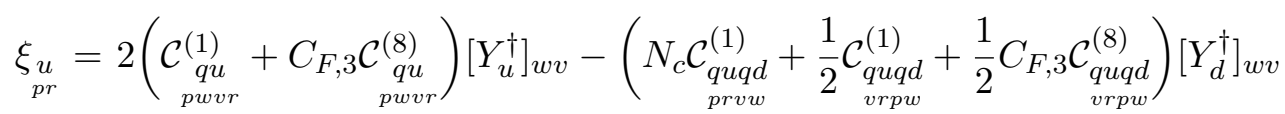

$$
\begin{aligned}
& +\underset{\text { lequp }}{(1)}\left[Y_{e}\right]_{w v}-\underset{\mathcal{C}_{\text {lnurp }}}{\mathcal{C}_{\text {vwr }}}\left[Y_{n}^{\dagger}\right]_{v w},
\end{aligned}
$$




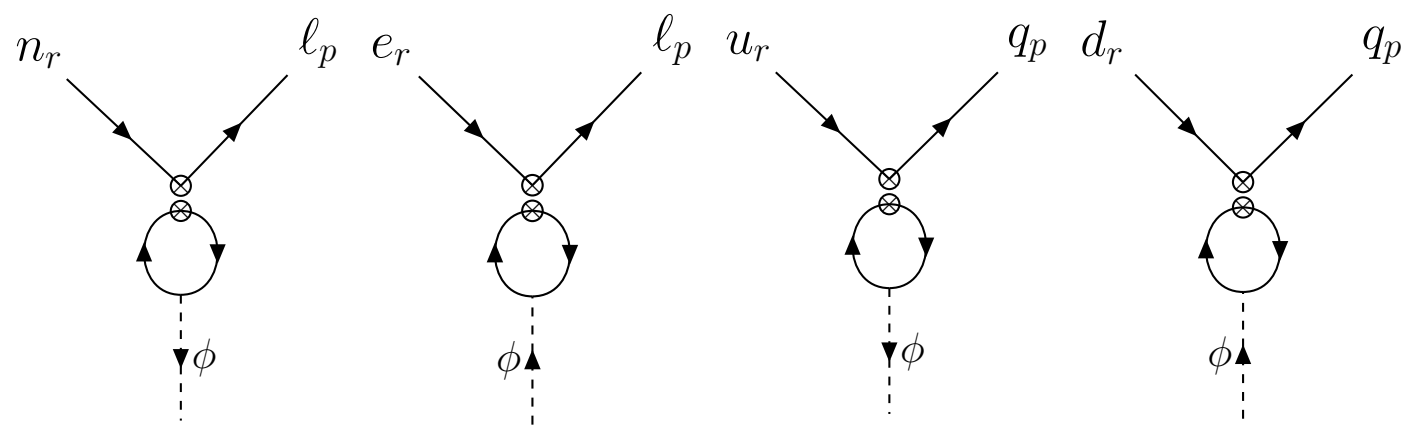

Figure 4. The Feynman diagrams associated with the $\xi$ parameters in eq. (2.16).

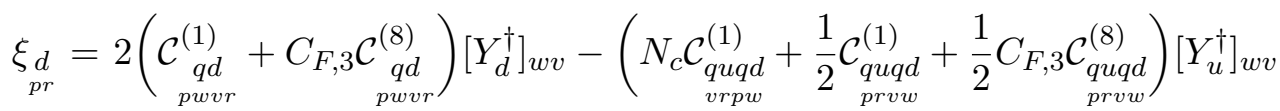

$$
\begin{aligned}
& -\mathcal{C}_{\text {ledq }}^{*}\left[Y_{e}^{\dagger}\right]_{v w}-\underset{\mathcal{C}_{\ell w q d}}{(1)}\left[Y_{n}\right]_{w v},
\end{aligned}
$$

where the quadratic Casimir $C_{F, 3}=\frac{4}{3}$ and the number of colors $N_{c}=3$. The $\xi$ parameter for right-handed neutrinos $n\left(\xi_{n}\right)$, corresponds to the new terms in SMNEFT, while the last terms in $\xi_{e}, \xi_{u}$ and $\xi_{d}$ are contributions from the right-handed neutrino Yukawa couplings not present in SMEFT.

\section{Results}

In this section, we present the Yukawa coupling contributions to the one-loop RGE for all four-fermion SMNEFT operators, and the new RGE terms for the four-fermion SMEFT operators due to the mixing between SMEFT and SMNEFT operators via the right-handed neutrino Yukawa couplings $Y_{n}$. The contributions from the fermionic operators come from the Feynman diagrams in figures 3(a) to 3(d), with contributions from figure 3(d) given by the $\xi$ parameters.

\subsection{Anomalous dimensions from Yukawa couplings: SMNEFT}

The bosonic operators in table 1 contribute to the SMNEFT ADM but not the SMEFT ADM. The contribution from the bosonic operator $\psi^{2} \phi^{2} D$ is shown in figure 3(e). The RGE of the dipole operators $\mathcal{O}_{\text {ln le }}$ and $\mathcal{O}_{\ell n q d}^{(3)}$ is modified by the $\psi^{2} X \phi$ operators in table 1 and the relevant diagrams are shown in figures $3(\mathrm{f})$ and $3(\mathrm{~g})$. These terms contain both gauge and Yukawa coupling contributions.

\subsection{1 $(\bar{R} R)(\bar{R} R)$}

$$
\begin{aligned}
& \dot{\mathcal{C}}_{p r d s}=-2\left[Y_{n} Y_{n}^{\dagger}\right]_{p r} \mathcal{C}_{\phi d}+2\left[Y_{d} Y_{d}^{\dagger}\right]_{s t} \mathcal{C}_{\phi n}-2\left[Y_{n}\right]_{p v}\left[Y_{n}^{\dagger}\right]_{w r} \mathcal{C}_{\text {vwst }}^{\ell d}-2\left[Y_{d}\right]_{s v}\left[Y_{d}^{\dagger}\right]_{w t} \mathcal{C}_{\text {vwn }}{ }_{\text {vwr }} \\
& -\left(\left[Y_{n}\right]_{p v}\left[Y_{d}\right]_{s w} \mathcal{C}_{\substack{\text { lnqd } \\
\text { vrwt }}}^{(1)}+\left[Y_{n}^{\dagger}\right]_{v r}\left[Y_{d}^{\dagger}\right]_{w t} \mathcal{C}_{\substack{\text { lnqd } \\
\text { vpws }}}^{(1) *}\right)
\end{aligned}
$$




$$
\begin{aligned}
& +12\left(\left[Y_{n}\right]_{p v}\left[Y_{d}\right]_{s w} \mathcal{C}_{\substack{\text { lnqd } \\
\text { vrwt }}}^{(3)}+\left[Y_{n}^{\dagger}\right]_{v r}\left[Y_{d}^{\dagger}\right]_{w t} \mathcal{C}_{\substack{\text { lnqd } \\
\text { vpws }}}^{(3) *}\right)
\end{aligned}
$$

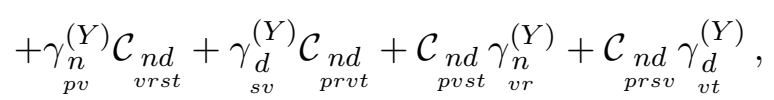

$$
\begin{aligned}
& \dot{\mathcal{C}}_{p r u}=-2\left[Y_{n} Y_{n}^{\dagger}\right]_{p r} \mathcal{C}_{\phi u}-2\left[Y_{u} Y_{u}^{\dagger}\right]_{s t} \mathcal{C}_{\phi n}-2\left[Y_{n}\right]_{p v}\left[Y_{n}^{\dagger}\right]_{w r} \mathcal{C}_{v w u}{ }_{v w t}-2\left[Y_{u}\right]_{s v}\left[Y_{u}^{\dagger}\right]_{w t} \mathcal{C}_{q u p r} \\
& +\left(\left[Y_{n}\right]_{p v}\left[Y_{u}^{\dagger}\right]_{w t} \mathcal{C}_{\text {lnuq }}+\left[Y_{n}^{\dagger}\right]_{v r}\left[Y_{u}\right]_{s w} \mathcal{C}_{\text {lnut }}^{*}\right)
\end{aligned}
$$

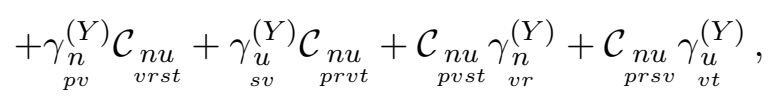

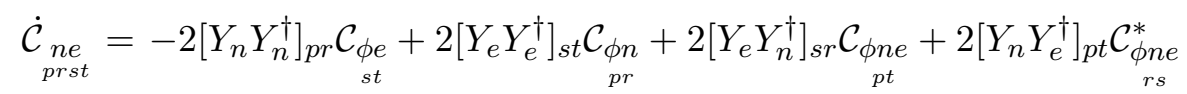

$$
\begin{aligned}
& -2\left[Y_{n}\right]_{p v}\left[Y_{n}^{\dagger}\right]_{w r} \mathcal{C}_{\text {le }}-\left(\left[Y_{n}\right]_{p v}\left[Y_{e}\right]_{s w} \mathcal{C}_{\text {lnle }}+\left[Y_{n}^{\dagger}\right]_{v r}\left[Y_{e}^{\dagger}\right]_{w t} \mathcal{C}_{\text {lnee }}^{*}\right) \\
& +\left(\left[Y_{n}\right]_{p w}\left[Y_{e}\right]_{s v} \mathcal{C}_{\text {lnle }}+\left[Y_{n}^{\dagger}\right]_{w r}\left[Y_{e}^{\dagger}\right]_{v t} \mathcal{C}_{\text {lnew }}^{*}\right)
\end{aligned}
$$

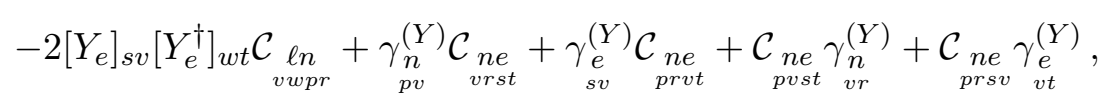

$$
\begin{aligned}
& \underset{p r s t}{\dot{\mathcal{C}}_{n n}}=-\left[Y_{n} Y_{n}^{\dagger}\right]_{p r} \mathcal{C}_{\phi n}-\left[Y_{n} Y_{n}^{\dagger}\right]_{s t} \mathcal{C}_{p n}-\left[Y_{n}\right]_{p v}\left[Y_{n}^{\dagger}\right]_{w r} \mathcal{C}_{v w}{ }_{v w s}-\left[Y_{n}\right]_{s v}\left[Y_{n}^{\dagger}\right]_{w t} \mathcal{C}_{v w n}{ }_{v w} \\
& +\gamma_{n}^{(Y)} \mathcal{C}_{v n}+\gamma_{v r s t}^{(Y)} \mathcal{C}_{n n}{ }_{p r v t}+\mathcal{C}_{p v s t} \gamma_{n r}^{(Y)}+\mathcal{C}_{p r s v} \gamma_{n t}^{(Y)}
\end{aligned}
$$

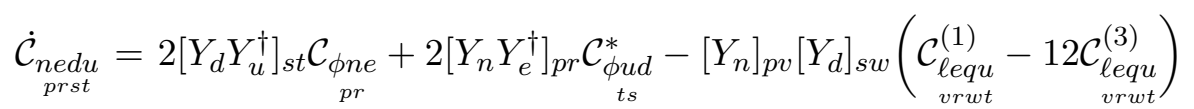

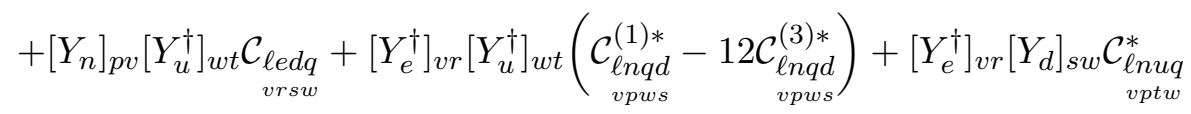

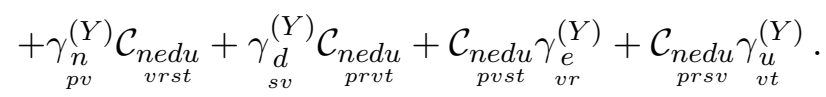

\section{$3.1 .2(\bar{L} L)(\bar{R} R)$}

$$
\begin{aligned}
& \dot{\mathcal{C}}_{p r s t}=\left[Y_{u}^{\dagger} Y_{u}-Y_{d}^{\dagger} Y_{d}\right]_{p r} \mathcal{C}_{\phi n}-2\left[Y_{n} Y_{n}^{\dagger}\right]_{s t} \mathcal{C}_{\phi q}^{(1)}-2\left[Y_{n}\right]_{s v}\left[Y_{n}^{\dagger}\right]_{w t} \mathcal{C}_{v w p r}^{(1)}-\left[Y_{u}\right]_{w r}\left[Y_{u}^{\dagger}\right]_{p v} \mathcal{C}_{\text {stuw }}{ }_{\text {stu }} \\
& -\left[Y_{d}\right]_{w r}\left[Y_{d}^{\dagger}\right]_{p v} \mathcal{C}_{\text {stvw }}+\frac{1}{2}\left(\left[Y_{n}\right]_{s w}\left[Y_{d}\right]_{v r} C_{\ell n q d}^{(1)}+\left[Y_{n t p v}^{\dagger}\right]_{w t}\left[Y_{d}^{\dagger}\right]_{p v} C_{\substack{\ell q d \\
w s r v}}^{(1) *}\right) \\
& +6\left(\left[Y_{n}\right]_{s w}\left[Y_{d}\right]_{v r} C_{\ell \text { lnqd }}^{(3)}+\left[Y_{n}^{\dagger}\right]_{w t}\left[Y_{d p v}^{\dagger}\right]_{p v} C_{\substack{\ell \text { wrd } \\
\text { wsr }}}^{(3) *}\right) \\
& -\frac{1}{2}\left(\left[Y_{n}\right]_{s w}\left[Y_{u}^{\dagger}\right]_{p v} C_{\substack{\ell u q q \\
w t v r}}+\left[Y_{n}^{\dagger}\right]_{w t}\left[Y_{u}\right]_{v r} C_{\substack{\text { lnuq } \\
\text { wsvp }}}^{*}\right)
\end{aligned}
$$

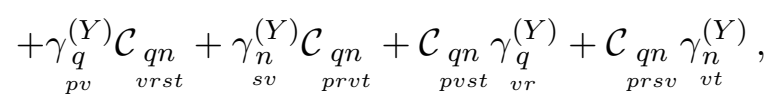

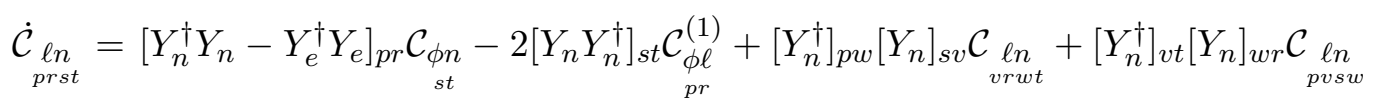




$$
\begin{aligned}
& -\left[Y_{e}^{\dagger}\right]_{p v}\left[Y_{e}\right]_{w r} \mathcal{C}_{s t v w}-2\left[Y_{n}^{\dagger}\right]_{p v}\left[Y_{n}\right]_{w r} \mathcal{C}_{v t s w}-2\left[Y_{n}^{\dagger}\right]_{p v}\left[Y_{n}\right]_{w r} \mathcal{C}_{v n n}{ }_{v w s t}-2\left[Y_{n}^{\dagger}\right]_{v t}\left[Y_{n}\right]_{s w} \mathcal{C} \underset{p v w r}{\ell \ell}
\end{aligned}
$$

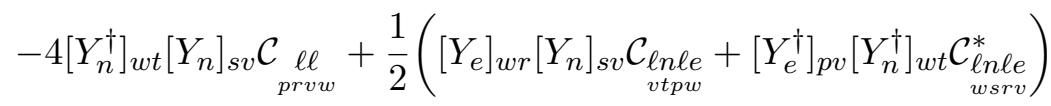

$$
\begin{aligned}
& +\left(\left[Y_{e}\right]_{w r}\left[Y_{n}\right]_{s v} \mathcal{C}_{p \text { lnle }}+\left[Y_{e}^{\dagger}\right]_{p v}\left[Y_{n}^{\dagger}\right]_{w t} \mathcal{C}_{l n \ell e v}^{*}\right)+\left[Y_{n}\right]_{s r} \xi_{p t}+\left[Y_{n}^{\dagger}\right]_{p t} \xi_{r s}^{*}+\underset{p v}{\gamma_{l}}(Y) \mathcal{C}_{v r s t} \\
& +\gamma_{n v}^{(Y)} \mathcal{C}_{\text {prvt }}+\mathcal{C}_{\text {pnst }} \gamma_{\text {pr }}^{(Y)}+\mathcal{C}_{\text {prs }} \gamma_{n t}^{(Y)}
\end{aligned}
$$

\subsection{3 $(\bar{L} R)(\bar{R} L)$ and $(\bar{L} R)(\bar{L} R)$}

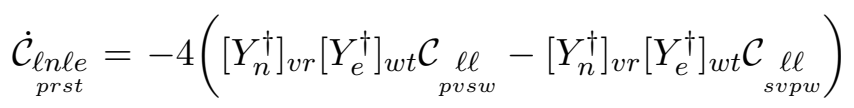

$$
\begin{aligned}
& +4\left(\left[Y_{n}^{\dagger}\right]_{w r}\left[Y_{e}^{\dagger}\right]_{v t} \mathcal{C}_{p v e}^{\ell \ell}-\left[Y_{n}^{\dagger}\right]_{w t}\left[Y_{e}^{\dagger}\right]_{v t} \mathcal{C} \underset{\text { sve }}{\ell \ell}\right) \\
& -4\left(\left[Y_{n}^{\dagger}\right]_{p v}\left[Y_{e}^{\dagger}\right]_{s w} \mathcal{C}_{\text {vrwt }}-\left[Y_{n}^{\dagger}\right]_{s v}\left[Y_{e}^{\dagger}\right]_{p w} \mathcal{C}_{\text {vrwt }}\right)+4\left[Y_{n}^{\dagger}\right]_{s w}\left[Y_{e}^{\dagger}\right]_{v t} \mathcal{C}_{p v w r} \\
& +4\left[Y_{n}^{\dagger}\right]_{v r}\left[Y_{e}^{\dagger}\right]_{p w} \mathcal{C}_{s v e t}+4 g_{1}\left(y_{e}+y_{\ell}\right) \mathcal{C}_{n B}\left[Y_{e}^{\dagger}\right]_{s t}-8 g_{1}\left(y_{e}+y_{\ell}\right) \mathcal{C}_{n B}\left[Y_{e r}^{\dagger}\right]_{p t} \\
& -6 g_{2} \mathcal{C}_{n W}\left[Y_{e}^{\dagger}\right]_{s t}+12 g_{2} \mathcal{C}_{n W}\left[Y_{e}^{\dagger}\right]_{p t}+4 g_{1}\left(y_{n}+y_{\ell}\right) \mathcal{C}_{e B}\left[Y_{n}^{\dagger}\right]_{p r} \\
& -8 g_{1}\left(y_{n}+y_{\ell}\right) \underset{p t}{\mathcal{C}_{e B}}\left[Y_{n}^{\dagger}\right]_{s r}-6 g_{2} \mathcal{C}_{e W}\left[Y_{n}^{\dagger}\right]_{p r}+12 g_{2} \mathcal{C}_{e W}\left[Y_{n t}^{\dagger}\right]_{s r}-\underset{p r}{2 \xi_{n}}\left[Y_{e}^{\dagger}\right]_{s t}
\end{aligned}
$$

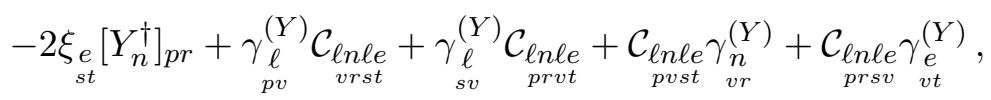

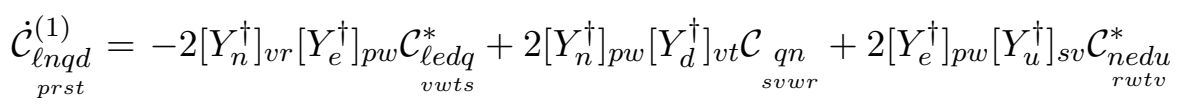

$$
\begin{aligned}
& +2\left[Y_{n}^{\dagger}\right]_{v r}\left[Y_{d}^{\dagger}\right]_{s w} \mathcal{C}_{p v w t}-2\left[Y_{d}^{\dagger}\right]_{w t}\left[Y_{u}^{\dagger}\right]_{s v} \mathcal{C}_{\text {lnuqu }}-2\left[Y_{n}^{\dagger}\right]_{p w}\left[Y_{d}^{\dagger}\right]_{s v} \mathcal{C}_{w r v t}
\end{aligned}
$$

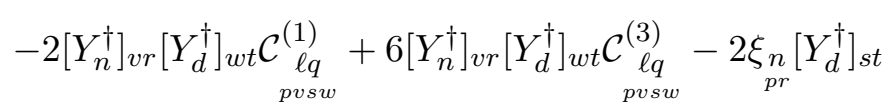

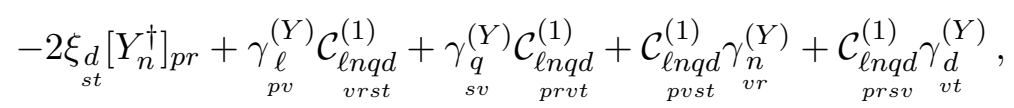

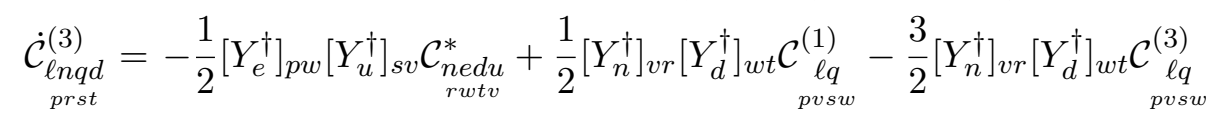

$$
\begin{aligned}
& \frac{1}{2}\left[Y_{n}^{\dagger}\right]_{v r}\left[Y_{d}^{\dagger}\right]_{s w} \mathcal{C}_{p v w t}+\frac{1}{2}\left[Y_{n}^{\dagger}\right]_{p w}\left[Y_{d}^{\dagger}\right]_{v t} \mathcal{C}_{\text {svw }}+\frac{1}{2}\left[Y_{n}^{\dagger}\right]_{p w}\left[Y_{d}^{\dagger}\right]_{s v} \mathcal{C}_{w d r t} \\
& -g_{1}\left(y_{d}+y_{q}\right) \underset{p r}{\mathcal{C}_{n B}}\left[Y_{d}^{\dagger}\right]_{s t}-g_{1}\left(y_{n}+y_{\ell}\right) \mathcal{C}_{d B}\left[Y_{n}^{\dagger}\right]_{p r}+\frac{3}{2} g_{2} \mathcal{C}_{n W}\left[Y_{d}^{\dagger}\right]_{s t} \\
& +\frac{3}{2} g_{2} \mathcal{C}_{d W}\left[Y_{n t}^{\dagger}\right]_{p r}+\underset{p v}{\gamma_{\ell}^{(Y)}} \mathcal{C}_{\text {lnqd }}^{(3)}+\underset{\text { vrst }}{\gamma_{q}^{(Y)}} \mathcal{C}_{\text {lnqd }}^{(3)}+\underset{\text { prvt }}{\mathcal{C}_{\text {lnqd }}^{(3)} \gamma_{n r}^{(Y)}}+\underset{\text { prs }}{(Y)}+\underset{\text { lnsv }}{(3)} \gamma_{d t}^{(Y)},
\end{aligned}
$$

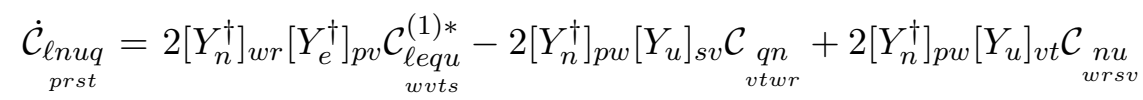

$$
\begin{aligned}
& +2\left[Y_{e}^{\dagger}\right]_{p w}\left[Y_{d}\right]_{v t} \mathcal{C}_{\text {nedus }}^{*}+2\left[Y_{n}^{\dagger}\right]_{v r}\left[Y_{u}\right]_{w t} \mathcal{C}_{\substack{\ell q w t \\
\text { rwo }}}^{(1)}+6\left[Y_{n}^{\dagger}\right]_{v r}\left[Y_{u}\right]_{w t} \mathcal{C}_{\substack{\ell q w t \\
\text { pqu }}}^{(3)}
\end{aligned}
$$




\begin{tabular}{|c|c|c|c|c|c|}
\hline \multicolumn{2}{|c|}{$(\bar{L} L)(\bar{L} L)$} & \multicolumn{3}{|c|}{$(\bar{L} L)(\bar{R} R)$} & \multicolumn{2}{|c|}{$(\bar{L} R)(\bar{R} L)$ and $(\bar{L} R)(\bar{L} R)$} \\
\hline $\mathcal{O}_{\ell \ell}$ & $\left(\bar{\ell}_{p} \gamma_{\mu} \ell_{r}\right)\left(\bar{\ell}_{s} \gamma^{\mu} \ell_{t}\right)$ & $\mathcal{O}_{\ell d}$ & $\left(\bar{\ell}_{p} \gamma_{\mu} \ell_{r}\right)\left(\bar{d}_{s} \gamma^{\mu} d_{t}\right)$ & $\mathcal{O}_{\ell e q u}^{(1)}$ & $\left(\bar{\ell}_{p}^{j} e_{r}\right) \epsilon_{j k}\left(\bar{q}_{s}^{k} u_{t}\right)$ \\
$\mathcal{O}_{\ell q}^{(1)}$ & $\left(\bar{\ell}_{p} \gamma_{\mu} \ell_{r}\right)\left(\bar{q}_{s} \gamma^{\mu} q_{t}\right)$ & $\mathcal{O}_{\ell u}$ & $\left(\bar{\ell}_{p} \gamma_{\mu} \ell_{r}\right)\left(\bar{u}_{s} \gamma^{\mu} u_{t}\right)$ & $\mathcal{O}_{\ell e q u}^{(3)}$ & $\left(\bar{\ell}_{p}^{j} \sigma_{\mu \nu} e_{r}\right) \epsilon_{j k}\left(\bar{q}_{s}^{k} \sigma^{\mu \nu} u_{t}\right)$ \\
$\mathcal{O}_{\ell q}^{(3)}$ & $\left(\bar{\ell}_{p} \gamma_{\mu} \tau^{I} \ell_{r}\right)\left(\bar{q}_{s} \gamma^{\mu} \tau^{I} q_{t}\right)$ & $\mathcal{O}_{\ell e}$ & $\left(\bar{\ell}_{p} \gamma_{\mu} \ell_{r}\right)\left(\bar{e}_{s} \gamma^{\mu} e_{t}\right)$ & $\mathcal{O}_{\ell e d q}$ & $\left(\bar{\ell}_{p}^{j} e_{r}\right)\left(\bar{d}_{s} q_{t}^{j}\right)$ \\
\hline & & $\mathcal{O}_{q u}^{(1)}$ & $\left(\bar{q}_{p} \gamma_{\mu} q_{r}\right)\left(\bar{u}_{s} \gamma^{\mu} u_{t}\right)$ & $\mathcal{O}_{q u q d}^{(1)}$ & $\left(\bar{\ell}_{p}^{j} n_{r}\right)\left(\bar{u}_{s} q_{t}^{j}\right)$ \\
& & $\mathcal{O}_{q u}^{(8)}$ & $\left(\bar{q}_{p} \gamma_{\mu} T^{A} q_{r}\right)\left(\bar{u}_{s} \gamma^{\mu} T^{A} u_{t}\right)$ & & \\
& & $\mathcal{O}_{q d}^{(1)}$ & $\left(\bar{q}_{p} \gamma_{\mu} q_{r}\right)\left(\bar{d}_{s} \gamma^{\mu} d_{t}\right)$ & & \\
& & $\mathcal{O}_{q d}^{(8)}$ & $\left(\bar{q}_{p} \gamma_{\mu} T^{A} q_{r}\right)\left(\bar{d}_{s} \gamma^{\mu} T^{A} d_{t}\right)$ & & \\
& & & \multicolumn{3}{|r}{} \\
\end{tabular}

Table 2. The 14 four-fermion SMEFT operators whose anomalous dimensions are modified by right-handed neutrino Yukawa couplings. Here, $I(A)$ is the adjoint index of $\mathrm{SU}(2)_{L}\left(\mathrm{SU}(3)_{C}\right)$.

$$
\begin{aligned}
& -2\left[Y_{n}^{\dagger}\right]_{v r}\left[Y_{u}\right]_{w t} \mathcal{C}_{\substack{\ell u \\
\text { psw }}}-2\left[Y_{u}^{\dagger}\right]_{s v}\left[Y_{d}\right]_{w t} \mathcal{C}_{\text {lnqd }}^{(1)}-\underset{p r v w}{2 \xi_{n}}\left[Y_{u}\right]_{s t}-2 \xi_{s t}^{*}\left[Y_{n}^{\dagger}\right]_{p r}
\end{aligned}
$$

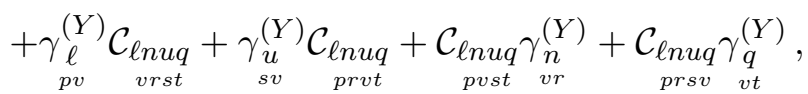

where $y_{n}=0, y_{e}=-1, y_{\ell}=-1 / 2, y_{d}=-1 / 3, y_{u}=2 / 3$, and $y_{q}=1 / 6$ are the hypercharges.

\subsection{Anomalous dimensions from Yukawa couplings: SMEFT}

The Yukawa interactions of the right-handed neutrinos modify the RGE of the four-fermion SMEFT operators listed in table 2. We only provide the additional terms induced by the right-handed neutrino Yukawa couplings $Y_{n}$. For the operators in the lower panel of table 2, the anomalous dimensions are modified via the $\xi$ parameters in eq. (2.16).

\subsection{1 $(\bar{L} L)(\bar{L} L)$}

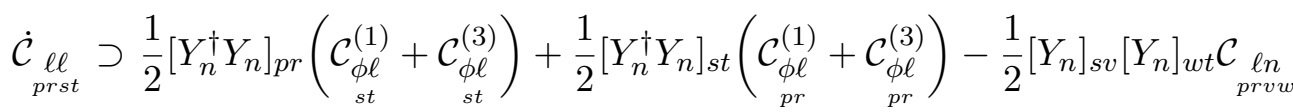

$$
\begin{aligned}
& -\frac{1}{2}\left[Y_{n}\right]_{p v}\left[Y_{n}\right]_{w r} \mathcal{C}_{\text {ln }}-\frac{1}{2}\left(\left[Y_{n}\right]_{v r}\left[Y_{e}\right]_{w t} \mathcal{C}_{\text {lnle }}+\left[Y_{n s w}\right]_{w t}\left[Y_{e}\right]_{v r} \mathcal{C}_{\substack{\ell \ell \ell e \\
\text { swp }}}\right) \\
& -\frac{1}{2}\left(\left[Y_{n}^{\dagger}\right]_{p v}\left[Y_{e}^{\dagger}\right]_{s w} \mathcal{C}_{l n l e}^{*}+\left[Y_{n}^{\dagger}\right]_{s w}\left[Y_{e}^{\dagger}\right]_{p v} \mathcal{C}_{\substack{\ell n \ell e \\
\text { twrv }}}^{*}\right)
\end{aligned}
$$

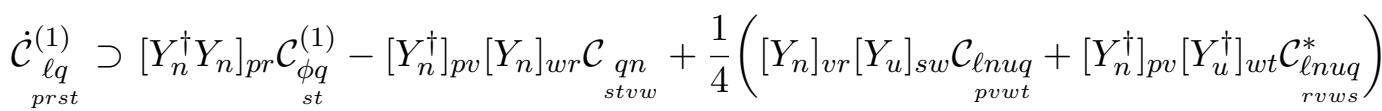

$$
\begin{aligned}
& -\frac{1}{4}\left(\left[Y_{n}\right]_{v r}\left[Y_{d}\right]_{w t} \mathcal{C}_{\text {lnqd }}^{(1)}+\left[Y_{n v s w}^{\dagger}\right]_{p v}\left[Y_{d}^{\dagger}\right]_{s w} \mathcal{C}_{\substack{l n q d \\
\text { rvtw }}}^{(1) *}\right) \\
& +3\left(\left[Y_{n}\right]_{v r}\left[Y_{d}\right]_{w t} \mathcal{C}_{\substack{\text { lnqd } \\
\text { pvsw }}}^{(3)}+\left[Y_{n}^{\dagger}\right]_{p v}\left[Y_{d}^{\dagger}\right]_{s w} \mathcal{C}_{\substack{\ell n q d \\
r v t w}}^{(3) *}\right)
\end{aligned}
$$




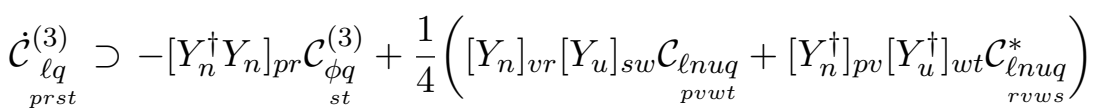

$$
\begin{aligned}
& +\frac{1}{4}\left(\left[Y_{n}\right]_{v r}\left[Y_{d}\right]_{w t} \mathcal{C}_{\substack{l n q d \\
\text { pvsw }}}^{(1)}+\left[Y_{n}^{\dagger}\right]_{p v}\left[Y_{d}^{\dagger}\right]_{s w} \mathcal{C}_{\substack{\text { lnqd } \\
r v t w}}^{(1) *}\right) \\
& -3\left(\left[Y_{n}\right]_{v r}\left[Y_{d}\right]_{w t} \mathcal{C}_{\substack{\text { lnqd } \\
\text { pvsw }}}^{(3)}+\left[Y_{n}^{\dagger}\right]_{p v}\left[Y_{d}^{\dagger}\right]_{s w} \mathcal{C}_{\text {lnqud }}^{(3) *}\right)
\end{aligned}
$$

\subsection{2 $(\bar{L} L)(\bar{R} R)$}

$$
\begin{aligned}
& \underset{\text { prst }}{\dot{\mathcal{C}}_{\ell d} \supset\left[Y_{n}^{\dagger} Y_{n}\right]_{p r} \mathcal{C}_{\phi d}-\left[Y_{n}\right]_{p v}\left[Y_{n}^{\dagger}\right]_{w r} \mathcal{C}_{\text {vd }}{ }_{\text {vwst }}}+\frac{1}{2}\left(\left[Y_{d}\right]_{s w}\left[Y_{n}\right]_{v r} \mathcal{C}_{\substack{\text { lnqd } \\
\text { pvwt }}}^{(1)}+\left[Y_{d}^{\dagger}\right]_{w t}\left[Y_{n}^{\dagger}\right]_{p v} \mathcal{C}_{\text {lnqd }}^{(1) *}\right) \\
& +6\left(\left[Y_{d}\right]_{s w}\left[Y_{n}\right]_{v r} \mathcal{C}_{\substack{l n q d \\
\text { pvwt }}}^{(3)}+\left[Y_{d}^{\dagger}\right]_{w t}\left[Y_{n}^{\dagger}\right]_{p v} \mathcal{C}_{\substack{\text { lnqd } \\
\text { rvws }}}^{(3) *}\right)
\end{aligned}
$$

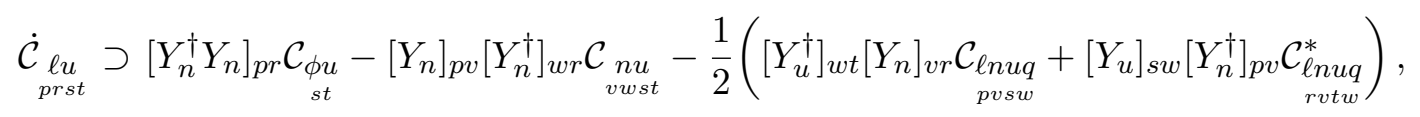

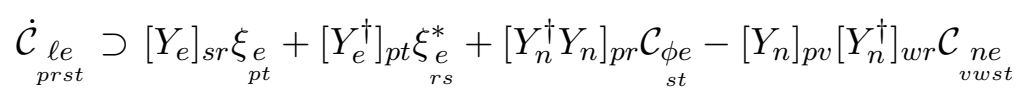

$$
\begin{aligned}
& +\frac{1}{2}\left(\left[Y_{e}\right]_{s w}\left[Y_{n}\right]_{v r} \mathcal{C}_{\text {lnle }}+\left[Y_{e}^{\dagger}\right]_{w t}\left[Y_{n}^{\dagger}\right]_{p v} \mathcal{C}_{\text {lnle }}^{*}\right)
\end{aligned}
$$

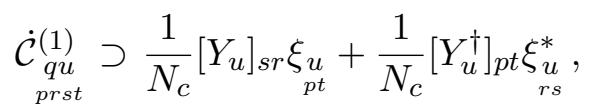

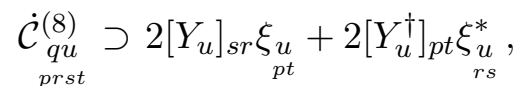

$$
\begin{aligned}
& \underset{p r s t}{\dot{\mathcal{C}}_{q d}^{(1)} \supset} \frac{1}{N_{c}}\left[Y_{d}\right]_{s r} \xi_{p t}+\frac{1}{N_{c}}\left[Y_{d}^{\dagger}\right]_{p t} \xi_{r s}^{*}, \\
& \underset{p r s t}{\dot{\mathcal{C}}_{q d}^{(8)}} \supset 2\left[Y_{d}\right]_{s r} \xi_{p t}+2\left[Y_{d}^{\dagger}\right]_{p t} \xi_{r s}^{*} .
\end{aligned}
$$

\subsection{3 $(\bar{L} R)(\bar{R} L)$ and $(\bar{L} R)(\bar{L} R)$}

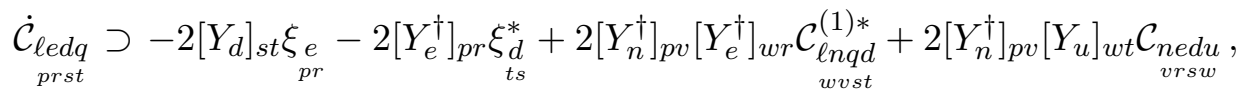

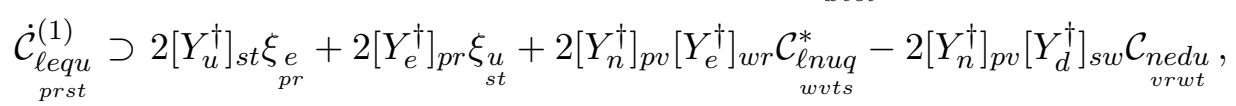

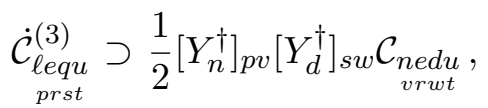

$$
\begin{aligned}
& \underset{\mathcal{C}_{q u s t}^{(1)}}{(1)} \supset-2\left[Y_{u}^{\dagger}\right]_{p r} \xi_{s t}-2\left[Y_{d}^{\dagger}\right]_{s t} \xi_{p r} .
\end{aligned}
$$

\section{Summary}

We presented the Yukawa terms of the one-loop anomalous dimension matrix for the dimension-six four-fermion operators of SMNEFT. This complements the gauge terms calculated in ref. [13]. Even if the right-handed neutrino Yukawa couplings are small, the 
induced mixings between SMNEFT operators can result in large RG running proportional to the other Yukawa couplings. The Yukawa couplings of the right-handed neutrinos also cause the SMEFT and SMNEFT operators to mix. We calculated the new ADM contributions for the 14 four-fermion SMEFT operators that are affected by this operator mixing.

\section{Acknowledgments}

This work was supported by NSF Grant No. PHY1915142 (A.D.), Humboldt Foundation (J.K.), DOE Grant No. DE-FG02-95ER40896 and PITT PACC (H.L.), and DOE Grant No. de-sc0010504 (D.M.).

Open Access. This article is distributed under the terms of the Creative Commons Attribution License (CC-BY 4.0), which permits any use, distribution and reproduction in any medium, provided the original author(s) and source are credited.

\section{References}

[1] W. Buchmüller and D. Wyler, Effective Lagrangian analysis of new interactions and flavor conservation, Nucl. Phys. B 268 (1986) 621 [InSPIRE].

[2] B. Grzadkowski, M. Iskrzynski, M. Misiak and J. Rosiek, Dimension-six terms in the Standard Model Lagrangian, JHEP 10 (2010) 085 [arXiv: 1008.4884] [INSPIRE].

[3] B. Henning, X. Lu and H. Murayama, How to use the Standard Model effective field theory, JHEP 01 (2016) 023 [arXiv: 1412.1837] [inSPIRE].

[4] I. Brivio and M. Trott, The Standard Model as an effective field theory, Phys. Rept. 793 (2019) 1 [arXiv: 1706. 08945] [INSPIRE].

[5] E.E. Jenkins, A.V. Manohar and M. Trott, Renormalization group evolution of the Standard Model dimension six operators. Part I. Formalism and $\lambda$ dependence, JHEP 10 (2013) 087 [arXiv: 1308.2627] [INSPIRE].

[6] E.E. Jenkins, A.V. Manohar and M. Trott, Renormalization group evolution of the Standard Model dimension six operators. Part II. Yukawa dependence, JHEP 01 (2014) 035 [arXiv: 1310.4838] [INSPIRE].

[7] R. Alonso, E.E. Jenkins, A.V. Manohar and M. Trott, Renormalization group evolution of the Standard Model dimension six operators. Part III. Gauge coupling dependence and phenomenology, JHEP 04 (2014) 159 [arXiv:1312.2014] [INSPIRE].

[8] F. del Aguila, S. Bar-Shalom, A. Soni and J. Wudka, Heavy Majorana neutrinos in the effective Lagrangian description: application to hadron colliders, Phys. Lett. B 670 (2009) 399 [arXiv: 0806.0876] [INSPIRE].

[9] A. Aparici, K. Kim, A. Santamaria and J. Wudka, Right-handed neutrino magnetic moments, Phys. Rev. D 80 (2009) 013010 [arXiv:0904.3244] [INSPIRE].

[10] S. Bhattacharya and J. Wudka, Dimension-seven operators in the standard model with right handed neutrinos, Phys. Rev. D 94 (2016) 055022 [Erratum ibid. 95 (2017) 039904] [arXiv: 1505.05264] [INSPIRE]. 
[11] Y. Liao and X.-D. Ma, Operators up to dimension seven in Standard Model effective field theory extended with sterile neutrinos, Phys. Rev. D 96 (2017) 015012 [arXiv:1612.04527] [INSPIRE].

[12] I. Bischer and W. Rodejohann, General neutrino interactions from an effective field theory perspective, Nucl. Phys. B 947 (2019) 114746 [arXiv:1905.08699] [INSPIRE].

[13] A. Datta, J. Kumar, H. Liu and D. Marfatia, Anomalous dimensions from gauge couplings in SMEFT with right-handed neutrinos, JHEP 02 (2021) 015 [arXiv: 2010.12109] [INSPIRE].

[14] M. Chala and A. Titov, One-loop running of dimension-six Higgs-neutrino operators and implications of a large neutrino dipole moment, JHEP 09 (2020) 188 [arXiv:2006.14596] [INSPIRE].

[15] T. Han, J. Liao, H. Liu and D. Marfatia, Scalar and tensor neutrino interactions, JHEP 07 (2020) 207 [arXiv: 2004.13869] [INSPIRE].

[16] M. Cvetič and P. Langacker, D-instanton generated Dirac neutrino masses, Phys. Rev. D 78 (2008) 066012 [arXiv:0803.2876] [INSPIRE]. 